\title{
Städte des Globalen Südens im Fokus von Klima- und Entwicklungsfinanz
}

\author{
Reregulierung, Disziplinierung und Depolitisierung
}

\author{
Monika Grubbauer \\ Hanna Hilbrandt
}

Dieser Aufsatz untersucht die Verschiebung von Finanzialisierungsprozessen unter dem Vorzeichen von klima- und entwicklungspolitischen Agenden auf das Terrain der Stadt. Mit der Fokussierung von Finanzmärkten auf ökologisch nachhaltige Projekte sowie deren Involvierung in entwicklungspolitische Handlungsstrategien hat sich das Engagement von Finanzmärkten nicht nur sektoral, sondern auch geographisch verbreitet und vertieft. Die globale Governance von Klima- und Entwicklungspolitik zielt verstärkt auf Städte in sogenannten „emerging markets“. Die damit einhergehenden organisatorischen, diskursiven und regulativen Verschiebungen werden in kritischen Teilen der Wirtschaftsgeographie (Bracking 2015a; Ouma/Johnson/Bigger 2018), der Entwicklungsforschung (Mader 2017; Mawdsley 2018) und der Nachhaltigkeitsforschung (Asiyanbi 2017) randständig auch ihrer städtischen Dimension diskutiert. Die neue Bedeutung der Stadt als Akteur, Objekt und Ort von klima- und entwicklungspolitischen Interventionen, die auf Vertiefung und Ausweitung von Kapitalflüssen aufbauen, ist jedoch in gegenwärtigen stadtpolitischen und Stadtforschungsdebatten noch nicht ausreichend erfasst.

Ziel unseres Beitrags istes, die beschriebenen Entwicklungen nachzuzeichnen und ihre Wirkung im Kontext finanzialisierter Stadtentwicklungsdynamiken herauszuarbeiten. Theoretisch nehmen wir Bezug auf Ansätze der politischen Ökonomie und politischen Ökologie, um zu beleuchten, inwiefern die Verknüpfung von klima- und entwicklungspolitischen Agenden auf dem Terrain der Stadt Prozesse der Reregulierung, Disziplinierung und Entpolitisierung befördert. Abschließend leiten wir daraus eine Agenda für zukünftige Forschung ab.

Ersteinreichung: 12. August 2019; Veröffentlichung online: 24. April 2020

An English abstract can be found at the end of the document.

\section{Einleitung}

Spätestens seit der globalen Finanzkrise von 2008 argumentieren breite Teile der Politikund der Finanzwirtschaft, dass drängende Problemlagenin Bezug auf Klimawandel und Armutsbekämpfung ohne die weitergehende Einbeziehung der Finanzmärkte nicht zu bewältigen seien.[1] In der Folge beobachtet die kritische Forschung zwei zunehmend verschränkte Entwicklungen: Zum einen die verstärkte Fokussierung von Finanzmärkten auf ökologisch nachhaltige Projekte; zum anderen deren Involvierung in entwicklungspolitische Strategien. Im Zuge beider Entwicklungen hat sich das Engagement von Finanzmärkten nicht nur sektoral, sondern auch geographisch verbreitet und vertieft. Beide Entwicklungen zielen insbesondere auf sogenannte, emerging markets , Niedrig und Mitteleinkommensländer, die als Wachstumsmärkte 
gelten (Carroll/Jarvis 2015). Dabei geraten insbesondere die Städte in den Fokus einer finanzialisierten Klima- und Entwicklungspolitik. Dieser Artikel untersucht, wie sich die gegenwärtige Neuausrichtung von globalen klima- und entwicklungspolitischen Handlungsfeldern auf dem Terrain der Stadt manifestiert, welche Konsequenzen sich abzeichnen und welcher Forschungsbedarf sich daraus für die kritische Stadtforschung ergibt.

Die Relevanz verstärkter Urbanisierungsprozesse sowie deren Herausforderungen und Chancen für die Klima- und Entwicklungspolitik sind weitgehend unumstritten (McGuirk/Bulkeley/Dowling 2016). Konsens globaler politischer Programme ist, dass gegenwärtige Klima- und Entwicklungskrisen in Städten zu lösen seien - vor allem in Städten Lateinamerikas, Asiens und Afrikas (UNEP 2013; Intergovernmental Panel on Climate Change 2014). Das 2015 verabschiedete urbane Ziel der UN-Agenda für nachhaltige Entwicklung und die Neue Urbane Agenda 2016 (United Nations 2016) werden auch wissenschaftlich weit rezipiert (Barnett/Parnell 2016, 2018; Caprotti et al. 2017; Satterthwaite 2017; Pieterse/Parnell/Haysom 2018). Demgegenüber weniger diskutiert werden die Bemühungen, Finanzmärkte wesentlich in die Umsetzung dieser Ziele einzubeziehen. Dabei sind die globalen Anstrengungen hinter dieser Agenda enorm: Schon vor Verabschiedung der Sustainable Development Goals (SDGs) hat die UN-Konferenz zur Entwicklungsfinanzierung (United Nations 2015) die Weichen für eine stärker finanzmarktorientierte Ausrichtung der Entwicklungspolitik gestellt. Aber auch die SDGs setzen in wesentlichen Punkten auf die Finanzwirtschaft (Mader 2017: 462; Weber 2017; Mitlin/Colenbrander/Satterthwaite 2018). Chancen und Möglichkeiten von Finanzmärkten in diesen Bereichen sind längst in Details ausbuchstabiert (Stern 2007; UNEP 2011; Zadek 2013).

Wir zeigen in diesem Artikel, wie sich die Verschneidung von globalen klima- und entwicklungspolitischen Agenden im städtischen Kontext exemplarisch manifestiert und argumentieren, dass die Verschränkung beider finanzpolitischer Handlungsfelder und ihre Fokussierung auf städtische Kontexte auch in der wissenschaftlichen Reflexion ein stärkeres Zusammendenken verschiedener disziplinärer Felder und Debatten erfordert. Wir bauen hierfür auf kritischen Debatten um die Finanzialisierung von Klima- und Entwicklungspolitik in Teilen der Wirtschaftsgeographie (Bracking 2015b; Ouma/Johnson/Bigger 2018), der Entwicklungsforschung (Mader 2017; Mawdsley 2018) und der Nachhaltigkeitsforschung (Asiyanbi 2017) auf. Dabei benennt die Literatur drei wesentliche Effekte der beschriebenen Entwicklungen, die fruchtbare Anknüpfungspunkte für die Stadtforschung bieten: Zu diesen Effekten gehört, erstens, ein erhöhter politischer Druck auf Nationalstaaten und Kommunen, den notwendigen regulatorischen Rahmen bereit zu stellen, wobei Städte als ,Partnerinnen“ eingebunden werden und die städtische Ebene vielfach als Testfall für weitergehende Reregulierungsprozesse dient (Roy 2010; Weber 2017). Damit einher geht, zweitens, die Disziplinierung von individuellem und staatlichem Handeln auf kommunaler Ebene durch die Ausrichtung an normativen Entwicklungszielen mit einer Vielzahl neuer Instrumente zur Bewertung kommunaler Projekte und Programme (Kopnina 2016; Briant Carant 2017; Weber 2017; Spann 2017). Die dritte Konsequenz der ,Urbanisierung' von finanzialisierter Klima- und Entwicklungspolitik besteht in der Depolitisierung 
der öffentlichen und fachlichen Debatten durch die Verschiebung von politischen Konflikten in den Bereich technischer Fragestellungen und die Umgehung demokratiepolitischer Prozesse (Rosol/Béal/Mössner 2017; Besedovsky 2018; Long/Rice 2018).

Im Anschluss an diese Debatten stellen wir die These auf, dass die Fokussierung von Klima- und Entwicklungsfinanz auf urbane Räume kommunale Akteure vor neue Herausforderungen stellt und sich deutliche Auswirkungen auf Stadtentwicklungsprozesse abzeichnen. Gleichzeitig konstatieren wir, dass gegenwärtige stadtpolitische und stadtforschungsbezogene Debatten die neue Bedeutung der Stadt als Akteur, Objekt und Ort in/von klimaund entwicklungspolitischen Interventionen, die auf Finanzmarktinklusion und der globalen Ausweitung von Kapitalflüssen aufbauen, noch nicht ausreichend erfassen. Um kritische Debatten der genannten Themen für die Stadtforschung zu erschließen und daraus eine Agenda für zukünftige Forschung abzuleiten, baut dieser Artikel auf einem ausführlichen Überblick über die relevante Literatur in Disziplinen und Zeitschriften auf, die bislang nur wenig in den Urban Studies und insbesondere innerhalb der deutschsprachigen Stadtforschung rezipiert wurden. Dies wird ergänzt durch empirische Befunde, die wir aus laufenden beziehungsweise gerade abgeschlossenen Forschungsprojekten ziehen. Dabei handelt es sich um Projekte zu neuen Instrumenten der Mikrofinanzierung in Mexiko (Grubbauer i. E.), zu grünen Kommunalanleihen (Hilbrandt/Grubbauer 2020) sowie zur City Creditworthiness Initiative der Weltbank (Grubbauer/Hilbrandt i. E.). Im Rahmen dieser Forschungsprojekte wurden Interviews mit Expert*innen in Entwicklungszusammenarbeit, Lokalbürokratie und Finanzwirtschaft durchgeführt, auf die wir uns in diesem Artikel punktuell beziehen.

Im Folgenden bilden wir zunächst die disziplinspezifischen Literaturdiskussionen zur Finanzialisierung von Klima und Entwicklung ab und reflektieren die neue Bedeutung von Städten des globalen Südens in diesen Entwicklungen. Zweitens diskutieren wir, wie diese Neuausrichtung globaler Nachhaltigkeits- und Entwicklungsagenden im Hinblick auf Prozesse der Reregulierung, Disziplinierung und Depolitisierung theoretisiert werden könnte. Drittens skizzieren wir eine Agenda für theoretische und empirische Erweiterungen der (deutschsprachigen) kritischen Stadtforschung im Bereich dieser Themenfelder.

\section{Städte des Südens im Fokus von Klima- und Entwicklungsfinanz}

Spätestens seit der Verabschiedung der UN-Agenda für nachhaltige Entwicklung (United Nations 2015) beobachten Autor*innen, wie ein breiter Konsens über die Bedeutung ökologischer Nachhaltigkeit durch immer weitere Kreise privater und politischer Akteure getragen und für finanzwirtschaftliche Agenden nutzbar gemacht wird (Rosol/Béal/Mössner 2017; Sullivan 2017). Auch in der Entwicklungsforschung beobachten kritische Autoren*innen, dass die klassische Partnerschaftszusammenarbeit durch eine neue Agenda ersetzt wird, in deren Zentrum das Konzept der financial inclusion steht, welches die Ausweitung von profitorientierten Finanzdiensten als Lösung für die Bekämpfung von Armut propagiert (Soederberg 2013; Carroll/ 
Jarvis 2015; Mader 2017). Die fortschreitende Urbanisierung von Armut mit wachsenden Zahlen von Stadtbewohnenden, die keinen Zugang zu formellen Arbeitsmärkten und angemessener sozialer und infrastruktureller Versorgung haben (Parnell/Pieterse 2010), sowie die Erkenntnis, dass Städte 60 bis 80 Prozent des weltweiten Energieverbrauchs und 75 Prozent der CO2Emissionen verursachen (UNEP 2011: 20), machen vor allem die schnell wachsenden Städte des globalen Südens zum zentralen Interventionsfeld finanzialisierter Klima- und Entwicklungspolitik (Pieterse 2011: 309).

Das neue Aufgabenverständnis der Rolle des Finanzmarktes im Hinblick auf Klima und Entwicklung beruht auch auf einer neuen Konzeptionierung der Rolle der Stadt und städtischer Verantwortung. Klimapolitik wird vermehrt auch auf städtische Räume bezogen und Stadtentwicklung damit ein wichtiges Handlungsfeld für Global Climate Finance (GCF, Entwicklungsfinanzierung, die im Einklang mit den Grundsätzen der Klimarahmenkonvention der Vereinten Nationen (UNFCCC) steht). Das zeigt sich an einer Reihe gut dotierter Programme, die darauf abzielen, Städte eng in die Lösung der Klimakrise einzubinden und ihnen hierbei zentrale Rollen zuzuweisen. Exemplarisch sind hier die von der GIZ (Deutsche Gesellschaft für Internationale Zusammenarbeit) geförderte Initiative FeliCity oder die Aktivitäten des Städtenetzwerks C40 im Rahmen der Climate Leadership Initiative und der Cities Finance Facility. Auch die neue Verantwortung des Finanzmarktes in der Armutsbekämpfung richtet sich nicht mehr ,nur', wie in der klassischen Entwicklungszusammenarbeit, auf ländliche Räume; vielmehr verschiebt finanzielle Inklusion Ideen und Diskurse und damit implizit auch Fragen der Verantwortung in Richtung Stadt. Dabei verändert sich neben der Problematisierung der Stadt als Verursacherin von Risiken und zentralem Ort der Intervention auch die Vorstellung der Möglichkeiten und Rollen von Städten in der Lösung dieser Krisen. Auch hier sind die oben genannten Aktivitäten von C40 exemplarisch: Städte des Südens sind darin nicht länger nur als Orte der Bekämpfung von Klimakrisen und Armut konzipiert, sondern auch Innovatorinnen im Sinne von Best Practice-Beispielen.

Dieses Kapitel beschreibt zentrale Entwicklungen, durch die sich Ideen verändern, Akteure an Macht gewinnen und Instrumente geschaffen werden, um ökologische Nachhaltigkeit und Entwicklungsziele im modernen Finanzmarktkapitalismus zu integrieren. Zum einen beobachten kritische Autoren*innen die Entstehung neuer Märkte durch die profitorientierte Verwertung ,ökologischer Nachhaltigkeit' durch die Finanzindustrie (Engels 2007; Pollard et al. 2008; Bracking 2012a; Fairbairn 2014; Feist/ Fuchs 2014; Dempsey/Suarez 2016; Asiyanbi 2017; Christophers 2018).Zum anderen verweisen aktuelle Debatten in der Entwicklungsforschung auf eine stärkere Durchdringung von entwicklungspolitischen Agenden mit Logiken, Instrumenten und Akteuren der Finanzindustrie (Weber 2004; Roy 2010; Jones 2012; Aitken 2013; Bond 2013; Carroll/Jarvis 2015; Isakson 2015; Mader 2015; Bracking 2016; Alami 2018; Harrison 2019). Wir zeigen im Folgenden auf, welche neuen Akteurskonstellationen und Neuerungen in den Politikinstrumenten mit dieser Neuausrichtung von Nachhaltigkeitsund Entwicklungsagenden auf globaler Ebene einhergehen und arbeiten im Anschluss daran heraus, wie die Stadt in diesen Prozessen - als zentraler 
Akteur, als Objekt der Intervention und als Ort der Profitgenerierung - an Bedeutung gewinnt.

\subsection{Neue Finanzakteure und Finanzinstrumente globaler Klima- und Entwicklungspolitik}

Die finanzmarktorientierte Neuausrichtung von Nachhaltigkeits- und Entwicklungsagenden geht mit neuen Aufgaben für öffentliche und privatwirtschaftliche Akteure sowie der Neuetablierung von Akteuren und der Neugründung von Netzwerken einher, baut aber auch auf der Einführung neuer Regulierungs- und Steuerungsinstrumente auf. Zusammen hat dies weitgehende Verschiebungen von Begriffsdefinitionen und letztlich auch Machtverhältnissen zur Folge.

In der green economy, die ökologische Modernisierung und Umweltinnovationen mit wirtschaftlichem Wachstum verbinden soll (Umweltbundesamt 2017), und konkret in den Projekten der GFC, gehören zu den neuen Akteuren Investor*innen, Verbände und Interessensvertretungen, Thinktanks, internationale Regierungsorganisationen, Klima-(I)NGOs, Verifizierungs- und Normenorganisationen, Expert*innen und viele mehr. Ähnliches lässt sich im Bereich der Entwicklungszusammenarbeit beobachten. Neben multilateralen Entwicklungsorganisationen, Wohltätigkeitseinrichtungen und Stiftungen rücken Akteure in den Vordergrund, die keinen wohlfahrtsstaatlichen Auftrag haben, etwa Kreditgeber und Kreditkartenunternehmen, große Banken, Unternehmensberatungen, Technologiefirmen, Mobilfunkanbieter und andere Akteure der Geschäftswelt. In beiden Politikfeldern verändern sich die Abläufe und Strukturen bestehender Entwicklungsorganisationen, vor allem solcher, die (neuerdings) als Finanzintermediäre fungieren. Dabei beobachtet die Literatur, wie Selbstverständnis und Aufgabenverteilung zwischen Weltbank, NGOs und staatlichen Akteuren vor allem im Globalen Süden stärker aneinanderrücken (Duffy 2006). Staatliche Akteure schaffen nicht länger nur die regulativen Rahmenbedingungen von Investitionen, vielmehr arbeiten Staat und (Finanz-)Wirtschaft gemeinsam an der Mobilisierung von (internationalem) Finanzkapital. So fördert beispielsweise das Programm des deutschen Bundesministeriums für Wirtschaftliche Zusammenarbeit und Entwicklung (BMZ) mit dem Namen develoPPP.de das Engagement von Investor*innen in den Bereichen, wo das Ministerium ,entwicklungspolitischen Handlungsbedarf' sieht.

Die Finanzialisierung von Nachhaltigkeits- und Entwicklungsagenden erfordert ein anspruchsvolles financial engineering und hat eine Reihe neuerer,nachhaltiger ${ }^{`}$ Technologien und Produktehervorgebracht. Im Bereich der green economy gehören zu den neueren Finanzierungsmechanismen grüne Kommunalanleihen (Hilbrandt/Grubbauer 2020), clean development mechanisms, also Mechanismen zur Treibhausgasreduktion (Bracking 2015a), CO2-Emissionsrechte (Engels 2007; MacKenzie 2009; McAfee 2016), Katastrophenanleihen (sogenannte, cat-bonds') (Johnson 2013) oder REDD+-Mechanismen (Pasgaard 2015). Auch die Umsetzung der Financial-Inclusion-Agenda geht mit der Entwicklung neuer Finanzinstrumente einher, die sich in Teilen mit den zuvor genannten überschneiden. Beispielsweise arbeitet die Entwicklungspolitik in den 
Bereichen Landwirtschaft, Gesundheit und Umwelt mit Katastrophenanleihen, Ebola oder vaccine bonds (Anleihen für die Finanzierung von Impfprogrammen), diaspora bonds (Anleihen, die sich besonders an im Ausland lebende Staatsangehörige richten, die Mittel auch in Krisenfällen ggf. nicht abziehen) oder index-based agricultural insurance, index-basierten Versicherungsfinanzmechanismen, die von Weltbank, Oxfam, UN und der Grameen Foundation propagiert werden, um klimabedingte Risiken von Ernteausfällen für Kleinbauern und Kleinbäuerinnen in Ländern des Südens zu mildern (Isakson 2015).

Mit den Veränderungen der Akteurslandschaft und der Politikinstrumente wandeln sich auch die grundlegenden Narrative über Klima und Entwicklung. Das wird insbesondere in der Neubenennung einer Reihe von Schlüsselbegriffen und einer Neubewertung von Orten und Räumen deutlich. Die begrifflichen Verschiebungen manifestieren sich in der Umbenennung von Entwicklungshilfe (foreign aid) in development finance, oder von Entwicklungsländern in emerging markets - Begriffe, die, wie Mader (2016) schreibt, die Wachstumsorientierung der entwicklungspolitischen Diskurse normalisieren. Es lässt sich auch erkennen, dass entwicklungspolitische Programme durch die Neuausrichtung der Förderstrukturen der Geberländer inhaltliche Veränderungen erfahren. Während der Fokus entwicklungspolitischer Programme noch vor zehn Jahren stark auf Governance und Dezentralisierung lag, kommen heute die größten Mittel aus ,Klimatöpfen‘. Für die Praxis der Entwicklungszusammenarbeit bedeutet dies, dass die Klimarelevanz aller Aktivitäten verstärkt belegbar und sichtbar gemacht werden muss (Interview 1).[2]

Gleichzeitig verändern sich auch Ideen über bestimmte Orte und deren Rolle in der Weltökonomie. Ehemalige Peripherien werden zu risikoreichen frontiers - Grenzräumen potentiell profitabler Investitionen (Mawdsley 2018), während Armutsbekämpfung zu Armutsmanagement oder zu „client ,welfare“" wird (Mader 2017: 472). Zudem verweist die Literatur auf Veränderungen in der Bedeutung verschiedener Maßstabsebenen (Bulkeley 2005; Pieterse/Parnell/Haysom 2018). Die Entwicklungsindustrie verbindet Mikro- und Makrobene durch den Anschluss geringverdienender Haushalte an die globale Finanzindustrie beziehungsweise die Integration von Mikrofinanz in globale Kapitalzirkulation (Roy 2010; Young 2010a, b). Mawdsley betont hierbei die Bedeutung von intermediären Akteuren, die zwischen diesen Ebenen verhandeln, etwa Aktienhändler*innen, Beratungsund Wirtschaftsprüfungsfirmen, aber auch lokale Multiplikator*innen, die die Rückzahlung von Mikrokrediten überwachen (Mawdsley 2018: 270). Auch Klimaprojekte verbinden Mikro- und Makroebene auf neue Weise, indem städtische Kommunen und Haushalte durch verändertes (Konsum-) Verhalten entscheidend zum Erreichen von Klimazielen beitragen sollen.

Zusammenfassend lässt sich feststellen, dass dem Finanzsektor Mitverantwortung für die Lösung der globalen ökologischen und entwicklungspolitischen Krise zugewiesen wird. Gleichzeitig verspricht dessen Engagement Wachstum und Profit (Besedovsky 2018: 25). Die historische Verantwortung der Industrieländer für den Klimawandel und die Zentralität des Verursacherprinzips, nach dem umweltrechtliche Verantwortlichkeit für Verunreinigungen dem Verursacher zugeschrieben wird, werden in 
Folge negiert. Laut Bracking (2015b) wird Verantwortung stattdessen an verantwortungsvolle Investitionen in ,nachhaltige' Geldanlagen der green economy geknüpft. Fragen danach, wie Märkte Ungleichheiten und Risiken generieren und auf Basis welcher Bedingungen Marktinklusion diese überwinden könnte, blendet die politische Debatte weitgehend aus (Carroll et al. 2019).

\subsection{Relevanz für die Stadtentwicklung}

Städte des globalen Südens gewinnen in sich verändernden MultilevelGovernance-Beziehungen in drei Aspekten an Bedeutung: als Akteure (als politische Institution und Stakeholder), Objekte (der Investition in die physisch-materiellen Infrastrukturen und Ökosysteme der Stadt) und als Orte (als institutionelle und räumliche Einheit) von und für klima- und entwicklungspolitische Interventionen und Investitionen.

Erstens werden Städte in sich wandelnden Akteurslandschaften wichtige Stakeholder und als solche auch aktiv als Partnerinnen angesprochen. Dabei wirken Städte zum einen als global-politische Akteure (Oosterlynck et al. 2019) auf internationaler Ebene mit. Dieser Bedeutungsgewinn hat sich zuletzt in der Verhandlung eines spezifischen städtischen UNNachhaltigkeitsziels (SDG11) gezeigt. Wie Barnett und Parnell (2018: 29) schreiben, konnten Städte in diesem Prozess aktiv ihre Interessen vertreten: "[T] he campaign for an Urban SDG involved the concerted mobilization of networks of local government and urban and regional planning, such as ICLEI (Local Government for Sustainability) and UCLG (United Cities and Local Governments), partnering with UN-level organisations such as Cities Alliance." In Zusammenarbeit mit anderen Netzwerken und städtischen Interessensvertretungen belegt die Durchsetzung eines städtischen Ziels die kollektive Handlungsmacht der städtischen Ebene. Aber auch die wachsende Macht einzelner, besonders globaler Städte in sogenannter „paradiplomacy“ (Setzer 2017: 504) wurde zuletzt auch in Klimafragen betont (Barber 2013). In diesen Kontexten tragen städtische Akteure selber aktiv zur Durchsetzung von Finanzialisierungsprozessen bei.

Zum anderen werden auch Sekundarstädte zunehmend als Partnerinnen globaler Klima- und Entwicklungsfinanz adressiert. An den Strategien der Weltbank und anderer entwicklungspolitischer Akteure - etwa der GIZ - lässt sich dies deutlich ablesen. Projekte wie die City Creditworthiness Initiative der Weltbank, industriepolitische Aktivitäten der UNIDO oder die Breathe Better Bond Initiative der IFC (International Finance Corporation, einer zur Weltbank gehörigen Entwicklungsbank) zielen darauf ab, Kommunen einzubinden und gleichzeitig deren Bedarfe im Hinblick auf konkrete Projekte und mögliche Finanzierungsmodelle abzufragen (Grubbauer/Hilbrandt i. E.). Damit einher gehen die Schulung städtischer Mitarbeiter*innen, das Bereitstellen von Wissen und Ressourcen und die Vernetzung der städtischen Akteure untereinander. Als Akteure erlangen Städte des globalen Südens jedoch auch durch die zunehmende Vernetzung durch Partnerschaftsnetzwerke - etwa ICLEI, 100 Resilient Cities, C40 - Sichtbarkeit und Bedeutung. Auch in diesen Initiativen ist das Anliegen zentral, Wissen zu verbreiten und Städte im Aufbau von Finanzinfrastruktur 
wesentlich zu unterstützen (Fünfgeld 2015). Aufseiten der Städte ergeben sich für die Verwaltungen aufwendige Lernprozesse, für die Ressourcen und politischer Wille mobilisiert werden müssen (Interview 1).

Zweitens erlangen Städte als Objekte Bedeutung, da physisch-materielle Infrastrukturen und städtische Ökosysteme verstärkt für Investitionen der globalen Klima- und Entwicklungsfinanz geöffnet werden. Die notwendigen Finanzinstrumente zur Erschließung von Stadtnatur sind in anderen Ökosystemen schon erprobt - etwa in der Finanzialisierung von Wäldern (To et al. 2012), Umweltschutz (Sullivan 2013), Land und Agrarwirtschaft (Ouma 2014, 2016) und Wasser (Reis 2017). Jedoch bezieht sich die Debatte um GCF nur randständig auf Stadtklima und Stadtnatur und spiegelt damit traditionelle Trennungen im Verständnis von Stadt und Natur (Heynen/ Kaika/Swyngedouw 2005). Ähnliches lässt sich in Entwicklungsdebatten konstatieren. Trotz einer dichten, kritischen Debatte um Finanzinklusion wird die zunehmende Verstädterung von Entwicklungsfragen nur mit Ausnahmen reflektiert. Die Fokussierung auf Städte des Südens als Objekte für die Anwendung von Finanzinstrumenten, die Nachhaltigkeits- und Entwicklungspolitiken im Hinblick auf Interessen des Nordens eng miteinander verschränkt, verweist auch auf ein Nord-Süd-Verhältnis, das historische Abhängigkeitsverhältnisse fortschreibt.

Der Handel mit Emissionsminderungsgutschriften baut darauf, Investitionen in die Ressourcen der Länder des globalen Südens zu ermöglichen. Entsprechend sind die in Policy-Kreisen viel zitierten ,Investitionshemmnisse ein zentraler Fokus aktueller Entwicklungsagenden (Scherer/Kramer 2019). So sollen etwa die mangelnde Verfügbarkeit von ausreichend gut vorbereiteten Projekten, höhere Investitionsrisiken und daher geringes Vertrauen in diese Märkte sowie deren begrenzte Kreditwürdigkeit durch entsprechende Instrumente überwunden werden. Zu solchen Politiken der Risikominimierung zählt auch die Mischfinanzierung von Projekten mit öffentlichen Zuschüssen, sogenanntes ,blending'. Dabei verbessern staatlich finanzierte Zuschüsse Finanzierungskonditionen und steigern die Rentabilität von Projekten, um privatwirtschaftliche Investitionen auch in sogenannten ,least developed countries' zu fördern (UNCDF 2018). Städtische Infrastrukturprojekte im Globalen Süden stellen hier ein zentrales Investitionsfeld dar.

Drittens sind Städte als Orte von Investitionen und Interventionen angesprochen, in denen, nachhaltige ' Technologien und Produkte und neue finanztechnische Instrumente implementiert werden. Dies richtet sich zunehmend auch auf Bereiche der Stadtentwicklung und weist insbesondere den Stadtverwaltungen eine neue Rolle als Finanzintermediäre und Partnerinnen von Finanzmarktakteuren zu. Dabei geht es nicht nur darum, dass Initiativen beispielsweise mit Mikrokrediten im informellen Wohnungsbau spezifische Problemlagen von städtischen Haushalten adressieren (Grubbauer i. E.). Vielmehr lässt sich beobachten, dass die subnationale Ebene auch die Funktionen übernimmt, mit Instrumenten zu experimentieren, um politische Interventionen oder praktische Engagements auszuarbeiten und zu testen. So gewinnen beispielsweise grüne Kommunalanleihen nicht nur an Bedeutung, um städtische Infrastruktur- und Bauprojekte zu initiieren. Die Stadt hat hier auch eine Vorreiterfunktion in der Emittierung dieser Anleihen. Städtische 
Emissionen erlauben es der nationalen Börse, technische und institutionelle Infrastrukturen zu etablieren, und potentiellen Investoren, Erfahrungen mit diesen Instrumenten zu sammeln (Interview 2).[3]

Zwar unterscheiden sich die neuen Produkte im Hinblick auf ihre finanztechnischen Details, gemeinsam ist ihnen aber, dass sie verschiedene Bereiche ökologischer und gesellschaftlicher Entwicklung marktförmig verwertbar machen, ohne dass die Wirksamkeit der Instrumente (langfristig) abgesichert ist. Dabei erschließt der Finanzsektor beispielsweise extreme Treibhausgas-Emissionen oder Wetterverhältnisse als Handelsgüter (Giamporcaro 2011: 121). Der CO2-Emissionshandel macht Profit mit Emissionsminderungsgutschriften, also mit Rechten, Treibhausgase zu emittieren, oder Gutschriften, die dadurch erworben werden, dass diese Gase nicht emittiert wurden (MacKenzie 2006). Entsprechend können beispielsweise grün zertifizierte Produkte Profite generieren, ohne dass deren Wert für die Bekämpfung des Klimawandels tatsächlich ermittelt, garantiert oder ein sogenannter ,green default ' geahndet werden könnte (Interview 2). Expert*innen aus der Praxis berichten zwar, dass aktuell mit einer Vielzahl von Nachhaltigkeitszertifizierungen experimentiert wird; die langfristigen Folgen von Projekten, die mit Geldern von Geberländern im Rahmen von Klimaprogrammen finanziert werden, bleiben jedoch weiterhin außerhalb des Betrachtungshorizontes (Interview 1).

Welche Möglichkeiten sich mit dem Fokus auf Städte des Südens als Akteure, Objekte und Orte von klima- und entwicklungspolitischen Investitionen für welche Städte eröffnen, bleibt zu untersuchen. Ob der damit verbundene Zuwachs an Ressourcen und (indirekter) Mitsprache dem Verantwortungsgewinn entspricht, ist eine offene Frage. Bereits jetzt konstatieren Autor*innen die mangelnde Beteiligung der Zivilgesellschaft an den besprochenen Entwicklungen beziehungsweise die zwiespältige Involvierung und Kooptierung von Aktivist*innen (Bracking 2015b). Um Fragen nach Machtverschiebungen und aus diesen folgende Ungleichheitsdynamiken zu erfassen, stellen wir im Folgenden Reregulierungs-, Disziplinierungs- und Depolitisierungsprozesse in den Vordergrund.

\section{Folgen und Probleme: Reregulierungs-, Disziplinierungs- und Depolitisierungsprozesse}

Aus der Verschneidung von globalen klima- und entwicklungspolitischen Agenden im städtischen Kontext ergeben sich für Städte des globalen Südens folgenschwere Veränderungen. Im Besonderen zeichnen sich Prozesse der Reregulierung lokalstaatlicher Rahmenbedingungen, der Disziplinierung von Stadtregierungen und der Depolitisierung von klima- und entwicklungspolitischen Debatten ab. Dieses Kapitel bietet Ansatzpunkte, diese Prozesse $\mathrm{zu}$ fassen und zu theoretisieren.

\subsection{Reregulierungsprozesse}

Die Finanzialisierung von Klima- und Entwicklungspolitik hat politischen Druck auf Nationalstaaten und Kommunen aufgebaut, die notwendigen Rahmenbedingungen zur Implementierung dieser Agenda bereitzustellen. 
Neben der Bereitstellung funktionierender materieller, technischer und institutioneller Infrastrukturen für Finanztransaktionen von Unternehmen und Haushalten ist die Umsetzung von Climate-Finance- und FinancialInclusion-Agenden daher mit regulatorischen Eingriffen verknüpft, die Investionen in klima- und entwicklungsbezogene Projekte in Ländern des Südens ermöglichen und fördern und so neue Märkte erschließen sollen.

Die für diese Aktivitäten notwendigen Reregulierungsprozesse stellen nationalstaatliche Souveränität zum einen durch die Übernahme einheitlicher globaler Normen in Frage. Zum anderen tragen sie aber auch dazu bei, bestehende verbindliche Normen zu unterlaufen und auszuhebeln (Bracking 2012b; Stark 2019). Dabei verschieben sich die Einflussbereiche nationalstaatlicher regulativer Intervention, privatwirtschaftlicher, rechtlich nicht bindender Normen und Standards und transnationaler Gesetzgebung; mit welcher Konsequenz dies geschieht, wird in der Literatur unterschiedlich diskutiert.

Die Weltbank nimmt in diesen Reregulierungsprozessen maßgeblich über drei zentrale Hebel Einfluss auf die Schuldnerländer: Sie interveniert über die kreditbasierte Finanzierung von Maßnahmen, zuletzt vor allem großer Infrastrukturprojekte, über Auflagen in Bezug auf die nationale Gesetzgebung zu Umwelt, Ressourcen und Eigentumsrechten sowie über Empfehlungen für entsprechende institutionelle Reformen. Goldman zeigt, wie diese drei Formen der Intervention verknüpft sind: ,[T] he development of fixed capital infrastructure (in this case, a joint-ventured hydroelectric facility) requires laws that establish certain property rights, which can only occur through the restructuring of state institutions“" (2001: 507). Soederberg (2013) betont die zentrale Rolle von marktbasierten Formen der Regulierung in der Ausweitung der Financial-Inclusion-Agenda. Sie argumentiert, dass rechtlich nicht bindendes soft law Einzelpersonen und Märkte durch seine informelle und ermessensabhängige Anwendung vor nationalstaatlicher Regulierung schützt und politisch sensible Bereiche der öffentlichen Anhörung und Verantwortung entzieht. Bracking unterstreicht demgegenüber insbesondere das Zusammenspiel von Nationalregierungen und Privatwirtschaft in der interessensgeleiteten Gestaltung der UN-Klimarahmenkonvention. Private Investmentfonds sieht sie als zentrale Knotenpunkte im Sinne einer „regulatory capture by powerful governments, corporates and the carbon trading industry, collectively organised within the institutional nodes of financialised private equity funds" (Bracking 2015b: 296). Die Rolle von Nationalstaaten in diesen Reregulierungsprozessen ist demnach widersprüchlich und umstritten, in jedem Fall aber hinsichtlich der Bedeutung und des Einflusses einzelner Staaten geographisch ungleich verteilt (Ouma/ Johnson/Bigger 2018: 505).

Die als Folge dieser Reregulierungs- und Vermarktlichungsprozesse entstehenden Standards und Zertifizierungssysteme sind widersprüchlich und die Existenz von Standards impliziert nicht notwendigerweise Standardisierung (Loconto/Busch 2010; Botzem/Dobusch 2012). Einerseits bestätigen Studien, dass die Kommodifizierung von Risiken weitreichende Reregulierungsprozesse erfordert (MacKenzie 2006; Giamporcaro 2011): Vermarktlichungsprozesse erfordern ein „making things the same“ (MacKenzie 2009: 440) und damit eine weitreichende Standardisierung von Produkten, die 
Rechtssicherheit auf internationalen Märkten herstellen soll. Dies geht mit der Entwicklung von kalkulativen Logiken einher, die vorgeben, soziale und ökologische Risiken so in Investitionsentscheidungen zu übersetzen, dass sie Prinzipien von Profitmaximierung und sozial-ökologischer Verantwortung integrieren (Giamporcaro 2011: 122). Die wichtigsten Beispiele für Ratingsysteme, bezogen auf klima- und entwicklungspolitische Ziele, sind heute das Global Impact Investing Reporting System (GIIRS), Standard IRIS, Global Impact Investing Network (GIIN) und United Nation's Principles for Responsible Investment. Andererseits ist die Wirksamkeit dieser Standards sehr unterschiedlich; vielfach konkurrieren unterschiedliche Standards um Aufmerksamkeit, Reichweite und Wirksamkeit (MacKenzie 2009; Ponte/ Cheyns 2013; Fortin 2018). Dazu kommt, dass globale und nationale Normen und Standards durch „portals of exception“ (Bracking 2016: 9) auch systematisch ausgehebelt werden. Dies nimmt, wie Bracking argumentiert, im Kontext der politischen Ökonomie vieler afrikanischer Länder Formen einer „commercialisation of souvereignity“ (ebd.: 11) an, in der lokale Eliten gemeinsam mit internationalen Investor*innen eine dem öffentlichen Interesse verpflichtete Rechtsprechung systematisch unterlaufen.

Für Städte stehen weitreichende Konsequenzen durch die beschriebenen Reregulierungsprozesse und den damit verbundenen Wandel von staatlicher Souveränität in Aussicht; einige werden bereits umgesetzt. Zum einen müssen Städte diese Prozesse mittragen und nationalstaatliche Veränderungen auf der kommunalen Ebene umsetzen. Bewertungsmaßstäbe und Indikatoren werden dabei oftmals von der nationalstaatlichen Ebene übernommen. Konkret sind Städte und Gemeinden dafür verantwortlich, förderfähige Projekte vorzubereiten und umzusetzen, und zwar so, dass Fördergelder gemäß den regulatorischen Vorgaben in Anspruch genommen werden können (siehe zum Beispiel die von der GIZ geförderte Initiative FeliCity, die zum Ziel hat, Kompetenzen der Kommunen in der Projektentwicklung und bei der Inanspruchnahme internationaler Finanzmittel zu stärken, siehe GIZ 2019). Vielfach geht damit einher, dass Städte gefordert sind, konsequente Entwicklungspläne aufzustellen, in die einzelne Projekte und damit auch Investitionen auf lange Zeit eingebettet werden, um Risiken für Finanzmarktakteure weiter zu minimieren. Zum anderen geraten Städte in den Fokus von Bemühungen, den nationalstaatlichen Rechtsrahmen durch Formen von soft laws gezielt zu umgehen. Das wird unterstützt durch ein sich herausbildendes Bewusstsein für die Bedeutung und potenzielle Reichweite städtischer Governance (Stren 2014; Obeng-Odoom 2017). Die Fokussierung auf die städtische Ebene als Hebel, um Reregulierungsprozesse in Gang zu setzen, spiegelt sich auch in den vielfältigen Initiativen von internationalen Entwicklungsinstitutionen, Städte als ,Partnerinnen` einzubinden.

\subsection{Disziplinierungsprozesse}

Der Bedeutungsgewinn von Städten im Kontext finanzialisierter Klima- und Entwicklungspolitik wirft Fragen nach der Disziplinierung von Stadtregierungen auf. Die beschriebene Ausrichtung des Handelns staatlicher Akteure und Haushalte an normativen Entwicklungszielen baut auf ,Wahrheiten“ über ökologisch und sozial nachhaltige Entwicklung (Kopnina 2016; Briant 
Carant 2017; Spann 2017; Weber 2017). Die SDGs geben hierfür seit 2015 den Deutungsrahmen vor. Dazu kommen die 2016 auf der Habitat-III-Konferenz verabschiedete New Urban Agenda (NUA) (Caprotti et al. 2017) sowie im deutschen Kontext die Empfehlungen des WBGU (Wissenschaftlicher Beirat der Bundesregierung Globale Umweltveränderungen) zur nachhaltigen Transformation der Städte (Hesse 2018). In der kritischen Debatte um globale Nachhaltigkeitsagenden werden die SDGs und andere Schlüsseldokumente und Rahmenpapiere als politische Konstrukte im Hinblick auf „ihnen zu Grunde liegende epistemologische, hegemoniale und polit-ökonomische Annahmen" (Gabay/Ilcan 2017: 337, Übers. d. A.) hinterfragt. Hervorgehoben wird in dieser Debatte die Verschiebung normativer Annahmen über Fortschritt und Entwicklung und deren Messbarkeit: „The SDGs lay out a post-millennium vision, presenting a radical shift in the normative position of governments on what constitutes universal human rights, where development should focus and how 'progress' will be measured." (Pieterse/ Parnell/Haysom 2018: 3) Darauf aufbauend stellt sich die Frage, wie sich individuelles und institutionelles Handeln durch die Ausrichtung an diesen Annahmen und daraus abgeleiteten Entwicklungszielen verändert.

Auf der Ebene von Individuen und Haushalten wird kritisch reflektiert, dass die Einführung neuer Finanzinstrumente mit weitreichenden Disziplinierungslogiken einhergeht. Für Mikrofinanzinstrumente gibt es diese Kritik schon seit langem, da die Vergabe von Mikrokrediten in Ermangelung anderer Sicherheiten an verhaltensbezogene Auflagen gebunden ist: Sparverhalten, Investitionsentscheidungen, Konsumverhalten und Haushaltsführung werden normiert und mit Regeln versehen, mit in Folge erhöhter Verantwortung und Belastung von Frauen im Haushalt (Rankin 2013; Kar/ Schuster 2016; Price 2019). Aber auch für andere Bereiche der Entwicklungspolitik, wie beispielsweise die Familienplanung, wird argumentiert, dass neue Formen der Ökonomisierung mit normativen Vorstellungen von Reproduktion einhergehen, wie Murphy (2017) am Beispiel der Invest-in a-Girl-Programme in Bangladesch deutlich macht. Im Rahmen der FinancialInclusion-Agenda kommt es zu einer Ausweitung derartiger Disziplinierungslogiken: Haushalte sollen nicht nur als Kleinunternehmen rational wirtschaften, sondern Finanzdienstleistungen in allen Bereichen des Lebens beanspruchen und dabei lernen, Risiken entsprechend abzuschätzen, wie Mader argumentiert: „People must show even more responsible and calculative behaviours than before: planning for and reacting to opportunities, needs, crises, setbacks, and so on, by making the right financial choices." (Mader 2017: 465) Ganz ähnliche Mechanismen gibt es auch in den Auflagen für GCF-Investitionen, wenn beispielsweise Kleinbauern und -bäuerinnen in der Auswahl ihrer Bepflanzung reglementiert werden, um Risikokalkulationen für Investor*innen gerecht zu werden (Goldman 2001; Borras et al. 2016).

Auch auf institutioneller und gesamtgesellschaftlicher Ebene werden Mechanismen der Disziplinierung deutlich. Insbesondere die ressourcenreichen und ökologisch diversen Länder des globalen Südens kommen hierbei in den Fokus einer sich verändernden ,Art des Regierens 'im Foucault'schen Sinn. Wie Goldman argumentiert, verpflichten die neuen Nachhaltigkeitsdiskurse sie moralisch zur Beteiligung an Umweltleistungen und öffnen gleichzeitig ihre Territorien durch entsprechende, rights regimes' für neue 
Formen der Vermessung, Kontrolle, Zertifizierung und Standardisierung (Goldman 2001: 499). Diese diskursiven Setzungen und regulatorischen Eingriffe verändern das Verhältnis von Subjekten und Umwelt grundlegend; vorausgesetzt werden nun Reflexivität und Handlungsfähigkeit, „,knowing“ and ,clarifying' one's relationship to nature and the environment as mediated through new institutions" (ebd.: 501). Entwicklungsorganisationen werden weitgehend dem neuen Paradigma verpflichtet und sind aktiv beteiligt, dafür den institutionellen und materiellen Rahmen zu schaffen. Disziplinierung kommt hierbei nicht nur ideologisch zum Tragen, sondern auch operativ durch die Implementierung neuer risikominimierender Managementansätze (Carroll/Jarvis 2014: 535).

Auch hinsichtlich der beschriebenen Formen der Disziplinierung von individuellem und institutionellem Handeln stellt sich die Relevanz für Städte als sehr hoch dar. So sind städtische Behörden wichtige Partner*innen in den transdisziplinären Förderformaten der internationalen Entwicklungsorganisationen und der Europäischen Union und passen ihre Strukturen an Förderbedingungen und Themen der Ausschreibungen an. Zudem entfalten neue Formen des Ratings und der Zertifizierung von Städten im Hinblick auf ihre Kreditwürdigkeit Wirkung, die weit über die bisherigen Städterankings, die typischerweise vor allem Image und Attraktivität von Städten in der Außendarstellung verbessern sollen, hinausgeht. Exemplarisch kann die City Creditworthiness Initiative der Weltbank genannt werden, die in Kooperation mit IFC, C40, Rockefeller Foundation und anderen Entwicklungsbanken durchgeführt wird. Diese Initiative schult Mitarbeiter*innen städtischer Behörden in 300 Städten in Niedrig- und Mitteleinkommensländern darin, die ,core creditworthiness challenges' der jeweiligen Städte - zentrale Herausforderungen auf dem Weg zur Erzielung von Kreditwürdigkeit - zu erkennen und zu überwinden. Damit sollen die , financial performance $\mathrm{der}$ kommunalen Haushalte verbessert und Zugriff auf Mittel privater Investor*innen zur Finanzierung von, climate-smart infrastructure and services gesichert werden (World Bank 2019). Es stellt sich die Frage, ob und welche Konsequenzen derartige Schulungen und Evaluationen, daraus abgeleitete Aktions- und Finanzpläne sowie neue Anforderungen der Zertifizierung nach globalen Standards in der realen Planungs- und Entwicklungspraxis haben.

\subsection{Depolitisierungsprozesse}

Die Verknüpfung städtischer Problemlagen mit den Logiken finanzmarktbasierter Klima- und Entwicklungspolitik führt vielfach zu einer Depolitisierung der Debatte. Zweifelsohne kommt es gerade im Bereich der Klimapolitik verstärkt zu politischen Protesten und zivilgesellschaftlichem Engagement. Die Rolle von Aktivist*innen wird in der Literatur jedoch kritisch diskutiert: Obwohl zivilgesellschaftliche Akteure kleine, repräsentative Gewinne verbuchen können, zieht beispielsweise Bracking den Schluss, dass sie als Teil von diesem Prozess „einer vertieften Praxis spätkapitalistischer Regierungsführung [advanced liberal governance] unterworfen werden“ (2015b: 281, Übers. d. A.). Neben solchen Prozessen der Kooptierung zeigt sich eine Depolitisierung von klima- und entwicklungspolitischen Fragen einerseits in der Verschiebung von politischen Konflikten in den Bereich technischer 
Fragestellungen; anderseits werden durch die Verschiebung von Klima- und Entwicklungsfinanz auf das Terrain der Stadt demokratiepolitische Prozesse auf der Ebene des Nationalstaats umgangen.

Erstens hat die Finanzialisierung von Klima- und Entwicklungspolitik Debatten in Richtung technischer Fragen verschoben (Besedovsky 2018) und massiv ausgeweitet. Wie Rosol et al. schreiben, hat der Begriff der Nachhaltigkeit, ob ökologisch oder sozial, sein kritisches Potential weitgehend eingebüßt: „Inzwischen werden sogar die deutsche Automobilproduktion, kanadische Ölpipelines in Alberta und Kernkraftwerke weltweit mit dem Hinweis auf Nachhaltigkeit und Klimaschutz politisch gerechtfertigt.“ (Rosol/Béal/ Mössner 2017: 1711, Übers. d. A.) Innerhalb des postpolitischen Regimes von nachhaltiger Governance dient der Fokus auf technische Detailfragen der globalen Klima- und Entwicklungsfinanzierung, beispielsweise rund um Emissionsreduktion und -handel, dazu, grundsätzliche politische Fragen auszublenden. Zivilgesellschaftliche Akteure hatten maßgeblich Anteil an der Entstehung der ersten globalen Nachhaltigkeitsnormen und -standards. Viele dieser Organisationen waren explizit dem Gedanken verpflichtet, die Beteiligung benachteiligter und betroffener Gruppen zu ermöglichen (Fortin 2018). Die heute relevanten Standardisierungsorganisationen, zum Beispiel das Intergovernmental Panel on Climate Change (IPCC), sehen sich nicht als politische Akteure, prägen politische Prozesse jedoch wirkmächtig (Loconto/Fouilleux 2013). Deren scheinbarer Fokus auf technische Lösungen erlaubt es, Ansprüche an Transparenz und Beteiligung weitestgehend auszublenden.

Ein zweiter Aspekt der Depolitisierung der Debatte um Klima- und Entwicklungsfinanzierung besteht in der Verlagerung politischer Prozesse auf das Terrain der Stadt. Dabei werden politische Entscheidungen und Verantwortung auf die lokalstaatliche Ebene delegiert. Manche Formen der Reregulierung, insbesondere durch rechtlich nicht bindende Standards und Normen, lassen sich auf dieser Ebene leichter realisieren; dazu kommt, dass angesichts von instabilen und teils dysfunktionalen politischen Systemen auf nationaler Ebene Städte des globalen Südens attraktive und vielfach handlungsfähigere Partner*innen darstellen. Die Verlagerung von klimaund entwicklungspolitischen Strategien vom Nationalstaat in die Stadt geht jedoch mit den oben beschriebenen Vermarktlichungsprozessen einher, in denen sich ,Marktrationalität' als zentrales Paradigma einschreibt (Carroll et al. 2019). Vielfach lassen fehlende Einnahmequellen in Verbindung mit Austeritätspolitiken Städten gar keine andere Wahl, als sich diesem Paradigma unterzuordnen und Finanzmarktakteure einzubinden. Carroll argumentiert, dass sich die vertieften Marktaktivitäten in der Entwicklungspolitik letzten Endes auch auf staatliche Strukturen auswirken. Das bleibt jedoch oft unerkannt und jenseits politischer Debatten. So hätte der IFC eine Schlüsselrolle „in deepening market activity around the state, while simultaneously fomenting shifts in the state that are seen as conducive to, ideallyconceived" patterns of capital accumulation" (Carroll 2012: 379, Herv. i. O.).

Abschließend stellt sich das Problem der Überprüfbarkeit der Effekte finanztechnischer Innovationen im Bereich von Klimaschutz und Armutsbekämpfung. Trotz der weitreichenden Öffnung der globalen klima- und entwicklungspolitischen Handlungsfelder für die Beteiligung des Finanzsektors 
sind die tatsächlich erzielten Lösungen und Verbesserungen oft ernüchternd. An vielen Stellen drängt sich der Befund auf, dass die Hinwendung finanzialisierter Klima- und Entwicklungspolitik zur Stadt (noch) nicht zur Lösung von Problemen beiträgt. Dennoch erfordert die Ausrichtung von Inhalten, behördlicher Praxis und institutionellen Strukturen an den neuen klima- und entwicklungspolitischen Agenden Zeit, Ressourcen und Expertise, die von möglicherweise wirkungsvolleren Projekten abgezogen werden. Das Framing von green investment als ,Spektakel“ lenkt davon ab, dass die technischen Mechanismen, die mit der green economy verbunden sind, im Hinblick auf tatsächliche Fortschritte durch ,grüne‘ Produkte kaum überprüfbar sind. Dazu kommt, dass die klima- und entwicklungsbezogenen Effekte für die Realisierung von Profiten nicht entscheidend sind. Obwohl Finanzmarktakteure Kontrolle über Investitionen erlangen, die Natur- und Umweltfragen betreffen, ist der daraus zu gewinnende Profit nicht ursächlich mit einem angemessenen Umstieg auf eine ökologisch nachhaltige Produktion und eine veränderte materielle Praxis verknüpft. Verschärft wird dies vielfach durch fehlende Transparenz; manche Maßnahmen werden einfach nicht umgesetzt, ohne dass dies Konsequenzen hätte (Bracking 2015a: 2353). Die Frage, welche neuen Hierarchien und Ausschlussmechanismen bei dieser Selektion zum Tragen kommen und wie sich diese mit bestehenden Machtgefällen zwischen Norden und Süden verschneiden, diskutieren wir im Fazit.

\section{Forschungsagenda und Fazit}

Trotz der Relevanz der aufgezeigten Entwicklungen steht eine kritische Debatte und Theoretisierung der Verschiebung von Finanzialisierungsprozessen unter dem Vorzeichen von klima- und entwicklungspolitischen Zielen auf das Terrain der Stadt vor allem in der deutschsprachigen Stadtforschung weiterhin aus. Mit diesem Aufsatz haben wir Brücken zu einer klima- und entwicklungspolitischen Literatur gebaut, die Finanzialisierungsprozesse in den Blick nimmt, um diese für den Ausbau bestehender Ansätze der Stadtforschung fruchtbar zu machen. Ziel war es einerseits, die klima- und entwicklungspolitischen Fragestellungen zu ,urbanisieren' und anderseits, Stadtforschungsthemen noch stärker an die Analyse der Wechselwirkungen zwischen ökonomischen, sozialen und ökologischen Faktoren im globalen Kapitalismus (Moore 2015; Brand/Wissen 2018; Patel/Moore 2018) anzuschließen, um Städte als zentrale Orte zu konzeptionalisieren, an denen finanzialisierte Klima- und Entwicklungspolitik ,gemacht` wird.

Für eine systematische Verknüpfung dieser Themenfelder bieten Debatten um nachhaltige Stadtforschung und städtische Governance wichtige Anknüpfungspunkte. Diese liegen im Bereich der theoriegeleiteten Debatten um nachhaltige Stadtentwicklung (Jong et al. 2015; McGuirk/Bulkeley/ Dowling 2016; Wachsmuth/Cohen/Angelo 2016; Rosol/Béal/Mössner 2017; Wachsmuth/Angelo 2018; Hajer/Versteeg 2019) und einer wachsenden Literatur zur Finanzialisierung städtischer Haushalte (Kirkpatrick 2016; Lauermann 2018; O’Brien/Pike 2018; Waldron 2018; Pike et al. 2019). Dazu kommen Theorieansätze, die den Zusammenhang von globalem Kapitalismus, Urbanisierung und der Inwertsetzung von Land, Natur und Ressourcen thematisieren (Geisler/Makki 2013; Li 2014; Arboleda 2015; Moore 2015; 
Knuth 2016; Dell'Angelo et al. 2017). Wichtige empirischeAnknüpfungspunkte für eine entsprechende Theoretisierung finden sich in Debatten um die Nachhaltigkeit einzelner Bauprojekte (Theurillat/Crevoisier 2013; Knuth 2016), ökologische Bewertungssysteme (Moore/Wilson 2009), Nachhaltigkeit als globales Wettbewerbsfeld (Jong et al. 2015; Rosol/Béal/Mössner 2017; Wachsmuth/Angelo 2018) und um die aktuelle Wachstumsagenda durch die Entwicklung neuer ,ökologischer` Technologien (Gibbs/Krueger/ Macleod 2013; Kitchin 2014; Caprotti/Springer/Harmer 2015; Datta 2015; Barthel 2016; Levenda 2019). Tendenziell verbleiben diese Debatten jedoch im Kontext abgegrenzter Stadtforschungsthemen und bilden die in diesem Aufsatz thematisierten Verschiebungen nicht im Kontext weiterer polit-ökonomischer Dynamiken ab. In diesem Fazit entwerfen wir eine empirische Forschungsagenda, die Forschungsbereiche der Stadtforschung stärker an Debatten um die Finanzialisierung von klima- und entwicklungspolitischen Agenden anschließt. Zudem skizzieren wir, wie eine theoretische Rahmung für diese empirischen Felder, die die beschriebenen Reregulierungs-, Disziplinierungs- und Depolitisierungsprozesse in Betracht zieht, aussehen könnte.

\subsection{Empirische und theoretische Herausforderungen für die Stadtforschung}

Der Bedeutungsgewinn von Städten als Akteure, Objekte und Orte finanzmarktbasierter klima- und entwicklungspolitischer Strategien hat - wie in Kapitel 3 diskutiert - wichtige Konsequenzen im Hinblick auf Fragen der Reregulierung, Disziplinierung und Depolitisierung.

Erstens werfen die Verschiebungen im Hinblick auf die Einflussbereiche transnationaler, nationalstaatlicher und privater beziehungsweise marktbasierter Regulierung und daraus resultierende Anforderungen an Städte wichtige Fragen auf. Empirisch zu untersuchen ist, wie Städte auf nationalstaatliche Vorgaben für Bewertungsmaßstäbe und Indikatoren reagieren, wie dies in Entwicklungsplänen und lokalen Projekten umgesetzt wird und welche Anpassungen im Hinblick auf Behördenstrukturen, Projektabwicklungen und politische Entscheidungsprozesse vorgenommen werden. Daran anschließend stellt sich die Frage, in welcher Weise und mit welchen Konsequenzen Städte in ihren Verwaltungen entsprechende Kapazitäten aufbauen (müssen), um nicht nur die Beantragung, Gewinnung und Implementierung von Projekten zu gewährleisten, sondern auch die Erfolge in Bezug auf Emissionsreduktion und Klimaanpassungsmaßnahmen nachweisbar und messbar zu machen. Dazu kommt die Frage nach der lokalen Varianz in der Implementierung der regulativen Vorgaben aufgrund von Übersetzungsprozessen zwischen internationaler, nationaler und lokaler Ebene oder auch aufgrund von Bemühungen, den nationalstaatlichen Rechtsrahmen gezielt zu unterlaufen und die städtische Ebene als Hebel zu nutzen, um Reregulierungsprozesse in Gang zu setzen und in einem institutionell und räumlich klar abgegrenzten Rahmen neue Finanzinstrumente zu testen.

Zweitens ergeben sich wichtige Fragen im Hinblick auf die Disziplinierung von individuellem und institutionellem Handeln, die empirisch noch weitgehend unerforscht sind. Diese betreffen die Wechselwirkungen zwischen 
neuen Formen des Ratings von Städten und der Zertifizierung städtischer Projekte auf der einen Seite und dem Handeln von Individuen und Institutionen auf der anderen Seite. Zu untersuchen ist, wie die Diskurse, die bei der Verschiebung klima- und entwicklungspolitischer Strategien auf das Terrain der Stadt wirksam werden, sich in Handlungs- und Disziplinierungslogiken manifestieren und in welcher Weise dies Alltagspraktiken in Institutionen und Haushalten verändert. Derartige akteursbezogene Forschungen könnten auch die bestehenden kritischen klima- und entwicklungspolitischen Forschungen bereichern, die sich vielfach auf policy- und diskursanalytische Verfahren beschränken und die Wirkmächtigkeit von Ideen und Diskursen oft nicht belegen können. Empirisch offen ist, an welcher Stelle und auf welcher Basis die Veränderungen von Managementpraktiken und Behördenstrukturen als positiv oder negativ zu bewerten sind. Dies betrifft insbesondere die Anpassung an Förderbedingungen und inhaltliche Setzungen von Ausschreibungen, mit denen sich Städte um Fördergelder bei internationalen Entwicklungsorganisationen und (supra-)staatlichen Institutionen bewerben. Wie die Projektgebundenheit der Wissenschaftsförderung die Zeithorizonte und Logiken behördlichen Handelns verändert, ist weitgehend unerforscht.

Das dritte thematische Feld für empirische Forschung ergibt sich aus den oben skizzierten Depolitisierungsprozessen. Im Zentrum steht hierbei die Frage, wie die neue Verantwortung von (manchen) Städten des globalen Südens und der Zuwachs finanzieller Ressourcen gleichzeitig mit Zwängen einhergeht, die politische Entscheidungsprozesse und demokratische Beteiligung einschränken. Eine offene Frage ist daher nicht nur, wie effektiv Klima- und Entwicklungsfinanz darin sind, Verbesserungen im Kontext der Klimakrise und Armutsbekämpfung zu erzielen und was die Konsequenzen eines Scheiterns in ökologischer oder sozialer Hinsicht wären; vielmehr stellt sich auch die Frage, wie der Raum für Kritik und Alternativen durch die hier beschriebenen Finanzialisierungsprozesse eingeschränkt wird. Eine entsprechende Forschungsagenda erfordert es, Wirksamkeit beziehungsweise Wirkungslosigkeit von Interventionen empirisch zu überprüfen und gegebenenfalls zu skandalisieren. Eine weitere wichtige Frage betrifft die Möglichkeiten der Mitsprache und Mitgestaltung für Städte als Partnerinnen in Projekten und Netzwerken mit internationalen Entwicklungs- und Finanzinstitutionen. Die neuen klima- und entwicklungspolitischen Investitionen in Städten sind hoch selektiv. Entscheidungen darüber, welche Investitionen mit welchen Zielgruppen und an welchen Orten priorisiert werden, sind weder transparent noch werden sie politisch diskutiert oder legitimiert. Empirisch wäre daher nicht nur zu untersuchen, welche Städte in welche Netzwerke eingebunden sind und wie dies neue Hierarchien zwischen Städten und Regionen schafft, sondern auch, wie Selektionsmechanismen gerechtfertigt oder depolitisiert werden. Hier wäre Forschung insbesondere jenseits der ,emerging markets wichtig, in Niedrigeinkommensländern mit Metropolen, die nicht in internationale Netzwerke und Partnerschaften eingebunden sind.

\subsection{Fazit}

Dieser Artikel hat skizziert, wie sich die Finanzialisierung von klima- und entwicklungspolitischen Agenden vermehrt in der Stadt manifestiert. Wir 
haben argumentiert, dass diese Verschiebung für die interdisziplinären Urban Studies dringende Fragen aufwirft, die vor allem die deutschsprachige Stadtforschung bislang nicht angemessen reflektiert. Unser Vorschlag ist, diese Verschiebungen vor allem in Hinblick auf Prozesse der Reregulierung, Disziplinierung und Depolitisierung zu untersuchen. Dieses Fazit verweist auf aus diesen Prozessen erwachsende Ungleichheitsdynamiken und entsprechend auf die Dringlichkeit und politische Relevanz der hier skizzierten Forschungsagenda. Abschließend heben wir die zentrale Rolle der interdisziplinären Urban Studies in der Beforschung dieser Agenda hervor.

Der zu beobachtende Fokus auf finanzialisierte Ansätze zur Lösung städtischer Herausforderungen im Bereich der Klima- und Entwicklungspolitik wirft dringende Fragen nach lokalen und globalen Ungleichheitsdynamiken auf. Auf lokaler Ebene führen Prozesse der Reregulierung, Disziplinierung und Depolitisierung zu einer Reihe sozio-materieller Veränderungen, die hier nur angerissen werden können: Wie beschrieben stehen die tatsächlichen Effekte der so finanzierten Klimaprojekte in Frage (Bond 2019; Hilbrandt/ Grubbauer 2020). Darüber hinaus beschreiben Stadtforscher*innen, wie Verdrängungen oder Umsiedlungen in der Folge von (ineffizienten) Klimaprojekten Ungleichheit verschärfen (Checker 2011). Entsprechend besteht für Silver (2017) die Brutalität des Klimawandels nicht nur in der sich entfaltenden sozial-ökologischen Krise, sondern auch in den Infrastrukturmaßnahmen, die im Namen von Klimaemissionsprojekten durchgeführt werden. Fragen der demokratischen Beteiligung unterrepräsentierter und vom Klimawandel vielerorts am stärksten betroffener Bevölkerungsgruppen werden in der Planung dieser Projekte vielfach außer Acht gelassen (Bulkeley et al. 2015; Fainstein 2015; Long/Rice 2018).

Auf globaler Ebene verschärfen die hier beschriebenen Entwicklungen Ungleichheitsdynamiken, vor allem entlang von Nord-Süd-Beziehungen. Zentral sind dabei Fragen nach der Wertigkeit von Wissensbeständen. Angesichts der Tendenz zur Erhebung metrischer, quantitativer Datenbestände nach Standards, die häufig in US-Amerika und Europa formuliert werden, und der Marginalisierung lokaler Wissensbestände - oft des globalen Südens - ist zu hinterfragen, zu welchem Zweck, nach welchen Grundannahmen und mit welchen Methoden wessen Wissen erhoben und welches Wissen produziert wird. Zudem stellen sich Fragen nach der Ungleichheit zwischen Städten. Schließlich bleibt die Auswahl an Städten, die als ,Partnerinnen“ für internationale Entwicklungsorganisationen an Bedeutung gewinnen, mehr oder weniger beschränkt. Obschon diese Städte an Mitsprache beim internationalen agenda setting gewinnen, fungieren sie auch als ,testbed' (Testballon) für die Übersetzung globaler Agenden auf die lokale Maßstabsebene. Dass hierbei ausgeschlossene Städte, die möglicherweise von ,klassischen“ Formen der Entwicklungszusammenarbeit profitieren konnten, nun „off the map“ (Robinson 2002) rücken, legt nahe, dass dieser Selektionsprozess globale Ungleichheitsverhältnisse weiter verschärft.

Obwohl die Urban Studies als interdisziplinäres Feld zentrale Forschungsperspektiven auf die beschriebenen Agenden verbinden, ergeben sich aus den Folgen der beschriebenen Veränderungen wesentliche Bedarfe nach einem Dialog zwischen angewandten und kritisch-theoretischen Feldern der Stadtforschung. Es lassen sich sowohl die mangelnde theoretische Fundierung 
spezialisierter, praxisorientierter Felder als auch die fehlende Rezeption realer politischer Entwicklungen und empirischer Zusammenhänge in der Theorieentwicklung konstatieren (Bunnell 2018; Grubbauer/Shaw 2018). Eine gemeinsame Untersuchung der politischen und der praxisbezogenen Fragen erfordert eine theoretische Öffnung angewandter Praxisfelder und eine Erschließung empirischen Wissens für die kritische Stadtforschung. Da es fraglich ist, ob kritische Stimmen jenseits des Policy-Mainstreams derzeit in Debatten um Klima- und Entwicklungsfinanzialisierung Gehör finden, bedarf eine entsprechende Agenda zudem neuer Formen der $\mathrm{Zu}$ sammenarbeit zwischen Wissenschaft und Politik. Aktuelle Erfahrungen in der Zusammenarbeit am research-policy interface (Patel et al. 2015; Mitlin/ Bartlett 2018; Pieterse/Parnell/Haysom 2018) verweisen sowohl auf die Möglichkeiten gegenseitigen Lernens als auch auf die Herausforderungen, die solchen Initiativen innewohnen (Lemanski 2018, Oldfield 2018), etwa entgegengesetzte Erwartungen an Zeithorizonte und Karriereanforderungen. Zentral wäre es nicht nur, Vereinnahmungen zu vermeiden, sondern auch entsprechend gewonnene Erkenntnisse, die über Beratung und gegenseitiges Voneinander-Lernen hinausgehen, in Politikdiskussionen einzubringen.

Da die hier vorgeschlagene Agenda soziale Ungleichheitsdynamiken zum Ausgangspunkt macht, bedarf es schließlich auch einer Auseinandersetzung mit der postkolonialen Kritik an Wissenshierarchien in den Urban Studies. Die andauernde Aufgabenverteilung der Stadtforschung, in der Theoriearbeit noch immer im globalen Norden geleistet wird und Theorien aus dem Süden beziehungsweise die weitreichenden Erfahrungen Forschender in Städten des globalen Südens vor allem im angloamerikanischen Publikationsraum kaum rezipiert werden, ist breit kritisiert worden. Dennoch sind wichtige Entwicklungen in Städten des globalen Südens weiterhin in einem ,Entwicklungsdiskurs' gefangen: Sie werden in den Zeitschriften dieser Themenbereiche publiziert und entsprechend in der internationalen Stadtforschungsdebatte nicht angemessen theoretisiert. Entwicklungen auch in südlichen Städten jenseits von ,Entwicklungsdiskursen` zu verstehen und in die Analyse einzubeziehen, ist nicht nur ein Gebot einer sich als ,global 'verstehenden Stadtforschung, sondern auch eine wichtige Voraussetzung, um verschärfte globale Ungleichheitsdynamiken adäquat zu erforschen.

\section{Endnoten}

[1] Die Arbeit, die dieser Publikation zugrunde liegt, wurde zum Teil durch das Programm P.R.I.M.E des Deutschen Akademischen Austauschdiensts (DAAD) mit Mitteln des Bundesministeriums für Bildung und Forschung (BMBF) finanziert.

[2] Berlin, 17.1.2020, GIZ.

[3] Mexiko Stadt, 24.7.2019, HR Rating. 


\section{Autor_innen}

Monika Grubbauer ist Professorin für Geschichte und Theorie der Stadt. Sie forscht zu urbanen Transformationsprozessen mit Fokus auf die Rolle von Architektur, Planung und Bauwirtschaft.

monika.grubbauer@hcu-hamburg.de

Hanna Hilbrandt ist Stadtgeographin und Architektin. Sie arbeitet zu Informalität, Wohnungspolitik, städtischer Governance und Finanzialisierung.

hanna.hilbrandt@geo.uzh.ch

\section{Literatur}

Aitken, Rob (2013): The Financialization of Micro-Credit. In: Development and Change $44 / 3,473-499$.

Alami, Ilias (2018): Money Power of Capital and Production of 'New State Spaces': A View from the Global South. In: New Political Economy 23/4, 512-529.

Arboleda, Martín (2015): Spaces of Extraction, Metropolitan Explosions: Planetary Urbanization and the Commodity Boom in Latin America. In: International Journal of Urban and Regional Research 40/1, 96-112.

Asiyanbi, Adeniyi P. (2017): Financialisation in the green economy: Material connections, markets-in-the-making and Foucauldian organising actions. In: Environment and Planning A 50/3, 531-548.

Barber, Benjamin (2013): If Mayors Ruled the World: Dysfunctional Nations, Rising Cities. New Haven: Yale University Press.

Barnett, Clive / Parnell, Susan (2016): Ideas, implementation and indicators. Epistemologies of the post-2015 urban agenda. In: Environment and Urbanization 28/1, 87-98.

Barnett, Clive / Parnell, Susan (2018): Spatial rationalities and the possibilities for planning in the New Urban Agenda for Sustainable Development. In: Gautam Bhan / Smita Srinivas / Vanessa Watson (Hg.), The Routledge Companion to Planning in the Global South. London/New York: Routledge, 25-36.

Barthel, Pierre-Arnaud (2016): Morocco in the era of eco-urbanism. Building a critical and operational research on an emerging practice in Africa. In: Smart and Sustainable Built Environment 5/3, 272-288.

Besedovsky, Natalia (2018): Finanzialisierung von Nachhaltigkeit. In: Sighard Neckel / Moritz Boddenberg / Martina Hasenfratz / Timo Wiegand / Natalia Besedovsky / Sarah Miriam Pritz (Hg.), Die Gesellschaft der Nachhaltigkeit - Umrisse eines Forschungsprogramms. Bielefeld: transcript, 25-40.

Bond, Patrick (2013): Debt, Uneven Development and Capitalist Crisis in South Africa: from Moody's macroeconomic monitoring to Marikana microfinance mashonisas. In: Third World Quarterly 34/4, 569-592.

Bond, Patrick (2019): Contradictory time horizons of Durban energy piping in an era of looming climate chaos. In: City 23/4-5, 631-645.

Borras, Saturnino M. / Franco, Jennifer C. / Isakson, S. Ryan / Levidow, Les / Vervest, Pietje (2016): The rise of flex crops and commodities: implications for research. In: The Journal of Peasant Studies 43/1, 93-115.

Botzem, Sebastian / Dobusch, Leonhard (2012): Standardization Cycles: A Process Perspective on the Formation and Diffusion of Transnational Standards. In: Organization Studies 33/5-6, 737-762.

Bracking, Sarah (2012a): How do Investors Value Environmental Harm/Care? Private Equity Funds, Development Finance Institutions and the Partial Financialization of Nature-based Industries. In: Development and Change 43/1, 271-293.

Bracking, Sarah (2012b): Secrecy jurisdictions and economic development in Africa: the role of sovereign spaces of exception in producing private wealth and public poverty. In: Economy and Society 41/4, 615-637.

Bracking, Sarah (2015a): Performativity in the Green Economy. How far does climate finance create a fictive economy? In: Third World Quarterly 36/12, 2337-2357.

Bracking, Sarah (2015b): The Anti-Politics of Climate Finance. The Creation and Performativity of the Green Climate Fund. In: Antipode 47/2, 281-302. 
Bracking, Sarah (2016): The Financialisation of Power. How Financiers Rule Africa. London/ New York: Routledge.

Brand, Ulrich / Wissen, Markus (2014): The financialisation of nature as crisis strategy. In: Journal für Entwicklungspolitik 2, 16-45.

Briant Carant, Jane (2017): Unheard voices: a critical discourse analysis of the Millennium Development Goals' evolution into the Sustainable Development Goals. In: Third World Quarterly 38/1, 16-41.

Bulkeley, Harriet (2005): Reconfiguring environmental governance: Towards a politics of scales and networks. In: Political Geography 24/8, 875-902.

Bulkeley, Harriet / Castán Broto, Vanesa / Edwards, Gareth A. S. (2015): An Urban Politics of Climate Change. Experimentation and the Governing of Socio-Technical Transitions. London/New York: Routledge.

Caprotti, Federico / Cowley, Robert / Datta, Ayona / Broto, Vanesa Castán / Gao, Eleanor / Georgeson, Lucien / Herrick, Clare / Odendaal, Nancy / Joss, Simon (2017): The New Urban Agenda. Key opportunities and challenges for policy and practice. In: Urban Research \& Practice 10/3, 367-378.

Caprotti, Federico / Springer, Cecilia / Harmer, Nichola (2015): „Eco“ For Whom? Envisioning Eco-urbanism in the Sino-Singapore Tianjin Eco-city, China. In: International Journal of Urban and Regional Research 39/3, 495-517.

Carroll, Toby (2012): Working On, Through and Around the State: The Deep Marketisation of Development in the Asia-Pacific. In: Journal of Contemporary Asia 42/3, 378-404.

Carroll, Toby / Gonzalez-Vicente, Ruben / Jarvis, Darryl S. L. (2019): Capital, conflict and convergence: a political understanding of neoliberalism and its relationship to capitalist transformation. In: Globalizations 16/6, 778-803.

Carroll, Toby / Jarvis, Darryl S. L. (2014): Introduction: Financialisation and Development in Asia under Late Capitalism. In: Asian Studies Review 38/4, 533-543.

Carroll, Toby / Jarvis, Darryl S. L. (2015): The New Politics of Development: Citizens, Civil Society, and the Evolution of Neoliberal Development Policy. In: Globalizations 12/3, 281-304.

Checker, Melissa (2011): Wiped Out by the „Greenwave“: Environmental Gentrification and the Paradoxical Politics of Urban Sustainability. In: City \& Society 23/2, 210-229.

Christophers, Brett (2018): Risking value theory in the political economy of finance and nature. In: Progress in Human Geography 42/3, 330-349.

Datta, Ayona (2015): New urban utopias of postcolonial India: „Entrepreneurial urbanization“ in Dholera smart city, Gujarat. In: Dialogues in Human Geography 5/1, 3-22.

Dell'Angelo, Jampel / D’Odorico, Paolo / Rulli, Maria Cristina / Marchand, Philippe (2017): The Tragedy of the Grabbed Commons: Coercion and Dispossession in the Global Land Rush. In: World Development 92, 1-12.

Dempsey, Jessica / Suarez, Daniel Chiu (2016): Arrested Development? The Promises and Paradoxes of „Selling Nature to Save It“. In: Annals of the American Association of Geographers 106/3, 653-671.

Duffy, Rosaleen (2006): Non-governmental organisations and governance states: The impact of transnational environmental management networks in Madagascar. In: Environmental Politics 15/5, 731-749.

Engels, Anita (2007): Market creation and transnational rule-making: The case of $\mathrm{CO}^{2}$ emissions trading. In: Marie-Laure Djelic / Kerstin Sahlin-Andersson (Hg.), Transnational governance: Institutional Dynamics of Regulation. Cambridge: Cambridge University Press, 329-348.

Fainstein, Susan (2015): Resilience and Justice. In: International Journal of Urban and Regional Research 39/1, 157-167.

Fairbairn, Madeleine (2014): „Like gold with yield“: evolving intersections between farmland and finance. In: The Journal of Peasant Studies 41/5, 777-795.

Feist, Marian / Fuchs, Doris (2014): Was heißt hier nachhaltig? In: Marcel Heires / Andreas Nölke (Hg.), Politische Ökonomie der Finanzialisierung. Wiesbaden: Springer VS, 225-240.

Fortin, Elizabeth (2018): Repoliticising multi-stakeholder standards processes: the Roundtable on Sustainable Biomaterials' standards and certification scheme. In: The Journal of Peasant Studies 45/4, 805-824.

Fünfgeld, Hartmut (2015): Facilitating local climate change adaptation through transnational municipal networks. In: Current Opinion in Environmental Sustainability 12, 67-73. 
Gabay, Clive / Ilcan, Suzan (2017): Leaving No-one Behind? The Politics of Destination in the 2030 Sustainable Development Goals. In: Globalizations 14/3, 337-342.

Geisler, Charles / Makki, Fouad (2013): People, Power, and Land: New Enclosures on a Global Scale. In: Rural Sociology 79/1, 28-33.

Giamporcaro, Stéphanie (2011): Sustainable and responsible investment in emerging markets: integrating environmental risks in the South African investment industry. In: Journal of Sustainable Finance \& Investment 1/2, 121-137.

Gibbs, David / Krueger, Rob / Macleod, Gordon (2013): Grappling with Smart City Politics in an Era of Market Triumphalism. In: Urban Studies 50/11, 2151-2157.

GIZ (2019): Financing Energy for Low-carbon Investment - Cities Advisory Facility (FELICITY). https://www.giz.de/de/weltweit/52753.html (letzter Zugriff am 4.8.2019).

Goldman, Michael (2001): Constructing an environmental state: Eco-governmentality and other transnational practices of a 'green' world bank. In: Social Problems 48/4, 499-523.

Grubbauer, Monika (i. E.): Assisted self-help housing in Mexico: advocacy, (micro)finance and the making of markets. In: International Journal for Urban and Regional Research.

Grubbauer, Monika / Hilbrandt, Hanna (i. E.): Urban shifts in the financialization of development: Learning to become creditworthy. In: Ève Chiapello / Anita Engels / Eduardo Gresse (Hg.), Financialisations of Development: Global Games and Local Experiments. London/New York: Routledge.

Grubbauer, Monika / Shaw, Kate (Hg.) (2018): Across Theory and Practice: Thinking through Urban Research. Berlin: Jovis.

Hajer, Maarten / Versteeg, Wytske (2019): Imagining the post-fossil city: why is it so difficult to think of new possible worlds? In: Territory, Politics, Governance 7/2, 122-134.

Harrison, Graham (2019): Authoritarian neoliberalism and capitalist transformation in Africa: all pain, no gain. In: Globalizations 16/3, 274-288.

Hesse, Markus (2018): Mobilising knowledge at the science-policy interface: „Humanity on the Move“. In: Monika Grubbauer / Kate Shaw (Hg.), Across Theory and Practice: Thinking Through Urban Research. Berlin: Jovis, 60-67.

Heynen, Nik / Kaika, Maria / Swyngedouw, Erik (2005): In the Nature of Cities. Urban Political Ecology and the Politics of Urban Metabolism. Florence: Taylor and Francis.

Hilbrandt, Hanna / Grubbauer, Monika (2020): Standards and SSOs in the contested widening and deepening of financial markets: The arrival of Green Municipal Bonds in Mexico City. In: Environment and Planning A: Economy and Space, Onlinefassung. https://doi.org/10.1177/0308518X20909391.

Intergovernmental Panel on Climate Change (2014): Climate Change 2014: Synthesis Report. Contribution of Working Groups I, II and III to the Fifth Assessment Report of the Intergovernmental Panel on Climate Change. https://www.ipcc.ch/site/assets/ uploads/2018/o2/AR5_SYR_FINAL_Front_matters.pdf (letzter Zugriff am 4.8.2019).

Isakson, S. Ryan (2015): Derivatives for Development? Small-Farmer Vulnerability and the Financialization of Climate Risk Management. In: Journal of Agrarian Change 15/4, 569-580.

Johnson, Leigh (2013): Catastrophe bonds and financial risk: Securing capital and rule through contingency. In: Geoforum 45, 30-40.

Jones, Branwen Gruffydd (2012): „Bankable Slums“: the global politics of slum upgrading. In: Third World Quarterly 33/5, 769-789.

Jong, Martin de / Joss, Simon / Schraven, Daan / Zhan, Changjie / Weijnen, Margot (2015): Sustainable-smart-resilient-low carbon-eco-knowledge cities; making sense of a multitude of concepts promoting sustainable urbanization. In: Journal of Cleaner Production 109, 25-38.

Kar, Sohini / Schuster, Caroline (2016): Comparative projects and the limits of choice: ethnography and microfinance in India and Paraguay. In: Journal of Cultural Economy 9/4, 347-363.

Kirkpatrick, L. Owen (2016): The New Urban Fiscal Crisis. In: Politics \& Society 44/1, 45-80.

Kitchin, Rob (2014): The real-time city? Big data and smart urbanism. In: GeoJournal 79/1, 1-14.

Knuth, Sarah (2016): Seeing Green in San Francisco: City as Resource Frontier. In: Antipode $48 / 3,626-644$.

Kopnina, Helen (2016): The victims of unsustainability: a challenge to sustainable development goals. In: International Journal of Sustainable Development \& World Ecology 23/2, 113-121. 
Lauermann, John (2018): Municipal statecraft. In: Progress in Human Geography 42/2, 205-224.

Lemanski, Charlotte (2018): Connecting research and policy: Individual and institutional triumphs and trials. In: Monika Grubbauer / Kate Shaw (Hg.), Across Theory and Practice: Thinking through Urban Research. Berlin: Jovis, 78-87.

Levenda, Anthony M. (2019): Thinking critically about smart city experimentation: entrepreneurialism and responsibilization in urban living labs. In: Local Environment 63/4, 1-15.

Li, Tania Murray (2014): What is land? Assembling a resource for global investment. In: Transactions of the Institute of British Geographers 39/4, 589-602.

Loconto, Allison / Busch, Lawrence (2010): Standards, techno-economic networks, and playing fields: Performing the global market economy. In: Review of International Political Economy 17/3, 507-536.

Loconto, Allison / Fouilleux, Eve (2013): Politics of private regulation: ISEAL and the shaping of transnational sustainability governance. In: Regulation \& Governance 8/2, 166-185.

Long, Joshua / Rice, Jennifer (2018): From sustainable urbanism to climate urbanism. In: Urban Studies 56/5, 992-1008.

MacKenzie, Donald (2006): An Engine not a Camera. How Financial Models Shape Markets. Cambridge, Massachusetts: MIT Press.

MacKenzie, Donald (2009): Making things the same: Gases, emission rights and the politics of carbon markets. In: Accounting, Organizations and Society 34/3-4, 440-455.

Mader, Philip (2015): The Political Economy of Microfinance: Financializing Poverty. Basingstoke: Palgrave Macmillan.

Mader, Philip (2016): Card Crusaders, Cash Infidels and the Holy Grails of Digital Financial Inclusion. In: Behemoth. A Journal on Civilisation 9/2, 50-81.

Mader, Philip (2017): Contesting Financial Inclusion. In: Development and Change 49/2, 461-483.

Mawdsley, Emma (2018): Development geography II: Financialization. In: Progress in Human Geography 42/2, 264-274.

McAfee, Kathleen (2016): Green economy and carbon markets for conservation and development: a critical view. In: International Environmental Agreements: Politics, Law and Economics 16/3, 333-353.

McGuirk, Pauline / Bulkeley, Harriet / Dowling, Robyn (2016): Configuring Urban Carbon Governance: Insights from Sydney, Australia. In: Annals of the American Association of Geographers 106/1, 145-166.

Mitlin, Diana / Bartlett, Sheridan (2018): Editorial: Co-production - key ideas. In: Environment and Urbanization 30/2, 355-366.

Mitlin, Diana / Colenbrander, Sarah / Satterthwaite, David (2018): Editorial: Finance for community-led local, city and national development. In: Environment and Urbanization 30/1, 3-14.

Moore, Jason W. (2015): Capitalism in the Web of Life: Ecology and the Accumulation of Capital. London/New York: Verso.

Moore, Steven A. / Wilson, Barbara B. (2009): Contested Construction of Green Building Codes in North America. The Case of the Alley Flat Initiative. In: Urban Studies 46/12, 2617-2641.

Murphy, Michelle (2017): The Economization of Life. Durham: Duke University Press.

Obeng-Odoom, Franklin (2017): Urban Governance in Africa Today: Reframing, Experiences, and Lessons. In: Growth and Change 48/1, 4-21.

O’Brien, Peter / Pike, Andy (2018): „Deal or no deal?“ Governing urban infrastructure funding and financing in the UK City Deals. In: Urban Studies 48/1, 1448-1476.

Oldfield, Sophie (2018): In everyday city struggles: Collaborative research and its inspirations. In: Monika Grubbauer / Kate Shaw (Hg.), Across Theory and Practice: Thinking through Urban Research. Berlin: Jovis, 223-231.

Oosterlynck, Stijn / Beeckmans, Luce / Bassens, David / Derudder, Ben / Segaert, Barbara / Braeckmans, Luc (Hg.) (2019): The City as a Global Political Actor. London/New York: Routledge.

Ouma, Stefan (2014): Situating global finance in the Land Rush Debate. A critical review. In: Geoforum 57, 162-166. 
Ouma, Stefan (2016): From financialization to operations of capital: Historicizing and disentangling the finance-farmland-nexus. In: Geoforum 72, 82-93.

Ouma, Stefan / Johnson, Leigh / Bigger, Patrick (2018): Rethinking the financialization of „nature“. In: Environment and Planning A 50/3, 500-511.

Parnell, Susan / Pieterse, Edgar (2010): The „Right to the City“: Institutional Imperatives of a Developmental State. In: International Journal of Urban and Regional Research 34/1, 146-162.

Pasgaard, Maya (2015): Lost in translation? How project actors shape REDD+ policy and outcomes in Cambodia. In: Asia Pacific Viewpoint 56/1, 111-127.

Patel, Raj / Moore, Jason W. (2018): A History of the World in Seven Cheap Things. A Guide to Capitalism, Nature, and the Future of the Planet. London/New York: Verso.

Patel, Zarina / Greyling, Saskia / Parnell, Susan / Pirie, Gordon (2015): Co-producing urban knowledge: experimenting with alternatives to 'best practice' for Cape Town, South Africa. In: International Development Planning Review 37/2, 187-203.

Pieterse, Edgar (2011): Recasting Urban Sustainability in the South. In: Development 54/3, 309-316.

Pieterse, Edgar / Parnell, Susan / Haysom, Gareth (2018): African dreams: locating urban infrastructure in the 2030 sustainable developmental agenda. In: Area Development and Policy 3/2, 149-169.

Pike, Andy / O’Brien, Peter / Strickland, Tom / Thrower, Graham / Tomaney, John (2019): Financialising City Statecraft and Infrastructure. Cheltenham UK u. a.: Edward Elgar Publishing.

Pollard, Jane S. / Oldfield, Jonathan / Randalls, Samuel / Thornes, John E. (2008): Firm finances, weather derivatives and geography. In: Geoforum 39/2, 616-624.

Ponte, Stefano / Cheyns, Emmanuelle (2013): Voluntary standards, expert knowledge and the governance of sustainability networks. Global Networks 13/4, 459-477.

Price, Sophia (2019): The risks and incentives of disciplinary neoliberal feminism: the case of microfinance. In: International Feminist Journal of Politics 21/1, 67-88.

Rankin, Katharine N. (2013): A critical geography of poverty finance. In: Third World Quarterly 34/4, 547-568.

Reis, Nadine (2017): Finance Capital and the Water Crisis: Insights from Mexico. In: Globalizations 14/6, 976-990.

Richardson, Tim / Cashmore, Matthew (2011): Power, knowledge and environmental assessment: the World Bank's pursuit of „good governance“. In: Journal of Political Power 4/1, 105-125.

Robinson, Jennifer (2002): Global and world cities: A view from off the map. In: International Journal of Urban and Regional Research 26/3, 531-554.

Rosol, Marit / Béal, Vincent / Mössner, Samuel (2017): Greenest cities? The (post-)politics of new urban environmental regimes. In: Environment and Planning A 49/8, 1710-1718.

Roy, Ananya (2010): Poverty Capital, Microfinance and the Making of Development. London/New York: Routledge.

Satterthwaite, David (2017): Successful, safe and sustainable cities: towards a New Urban Agenda. In: Commonwealth Journal of Local Governance 19, 3-18.

Scherer, Nora / Kramer, Anja Nina (2019): Blending 2.0: Mobilisierung privaten Kapitals auch in armen Ländern? https://www.kfw-entwicklungsbank.de/PDF/Download-Center/ PDF-Dokumente-Development-Research/2019_04_17_EK_Blending_Mobilisierung_ privaten_Kapitals_DE.pdf (letzter Zugriff am 5.5.2019).

Setzer, Joana (2017): How Subnational Governments are Rescaling Environmental Governance: The Case of the Brazilian State of São Paulo. In: Journal of Environmental Policy \& Planning 19/5, 503-519.

Silver, Jonathan (2017): The climate crisis, carbon capital and urbanisation: An urban political ecology of low-carbon restructuring in Mbale. In: Environment and Planning A 49/7, 1477-1499.

Soederberg, Susanne (2013): Universalising Financial Inclusion and the Securitisation of Development. In: Third World Quarterly 34/4, 593-612.

Spann, Michael (2017): Politics of Poverty: The Post-2015 Sustainable Development Goals and the Business of Agriculture. In: Globalizations 14/3, 360-378.

Stark, Johanna (2019): Law for Sale: A Philosophical Critique of Regulatory Competition. Oxford: Oxford University Press. 
Stern, Nicholas (2007): The Economics of Climate Change. Cambridge: Cambridge University Press.

Stren, Richard (2014): Urban Service Delivery in Africa and the Role of International Assistance. In: Development Policy Review 32/1, 19-37.

Sullivan, Sian (2013): Banking Nature? The Spectacular Financialisation of Environmental Conservation. In: Antipode 45/1, 198-217.

Sullivan, Sian (2017): Making nature investable: from legibility to leverageability in fabricating „nature“ as „natural capital“. In: Science and Technology Studies 31/3, 47-76.

Theurillat, Thierry / Crevoisier, Olivier (2013): The Sustainability of a Financialized Urban Megaproject. The Case of Sihlcity in Zurich. In: International Journal of Urban and Regional Research 37/6, 2052-2073.

To, Phuc Xuan / Dressler, Wolfram H. / Mahanty, Sango / Pham, Thu Thuy / Zingerli, Claudia (2012): The Prospects for Payment for Ecosystem Services (PES) in Vietnam: A Look at Three Payment Schemes. In: Human Ecology: an Interdisciplinary Journal 40/2, 237-249.

Umweltbundesamt (2017): Übergang in eine Green Economy: Systemische Hemmnisse und praktische Lösungsansätze. https://www.umweltbundesamt.de/sites/default/files/ medien/1410/publikationen/2017-11-21_uib_02-2017_green-economy_v2.pdf (letzter Zugriff am 7.8.2019).

UNCDF (2018): Blended Finance in the Least Developed Countries. The State of Blended Finance. https://www.uncdf.org/article/4220/blended-finance-in-ldcs-report (letzter Zugriff am 18.7.2019).

UNEP (2011): Towards a green economy: pathways to sustainable development and poverty eradication - A synthesis for policy makers. https://sustainabledevelopment.un.org/ index.php?page $=$ view\&type $=400 \& n r=126 \&$ menu $=35$ (letzter Zugriff am 4.8.2019).

UNEP (2013): City-level decoupling: urban resource flows and the governance of infrastructure transitions. http://www.unep.org/resourcepanel/portals/24102/pdfs/ Cities-Full_Report.pdf (letzter Zugriff am 4.8.2019).

United Nations (2015): Addis Abeba Action Agenda. https://sustainabledevelopment. un.org/index.php?page $=$ view\&type $=400 \& n r=2051 \&$ menu $=35$ (letzter Zugriff am 9.8.2019).

United Nations (2016): New Urban Agenda. Deutsche Übersetzung. http://habitat3.org/ wp-content/uploads/NUA-German.pdf (letzter Zugriff am 24.3.2020).

Wachsmuth, David / Angelo, Hillary (2018): Green and Gray: New Ideologies of Nature in Urban Sustainability Policy. In: Annals of the American Association of Geographers 108/4, 1038-1056.

Wachsmuth, David / Cohen, Daniel Aldana / Angelo, Hillary (2016): Expand the frontiers of urban sustainability. In: Nature 536/7617, 391-393.

Waldron, Richard (2018): Capitalizing on the State: The political economy of Real Estate Investment Trusts and the „Resolution“ of the crisis. In: Geoforum 90, 206-218.

Weber, Heloise (2004): The „New Economy“ and Social Risk: Banking on the Poor? In: Review of International Political Economy 11/2, 356-386.

Weber, Heloise (2017): Politics of „Leaving No One Behind“: Contesting the 2030 Sustainable Development Goals Agenda. In: Globalizations 14/3, 399-414.

World Bank (2019): City Creditworthiness Initiative: A Partnership to Deliver Municipal Finance https://www.worldbank.org/en/topic/urbandevelopment/brief/ city-creditworthiness-initiative (letzter Zugriff am 9.8.2019).

Young, Stephen (2010a): Gender, Mobility and the Financialisation of Development. In: Geopolitics 15/3, 606-627.

Young, Stephen (2010b): The „Moral Hazards“ of Microfinance: Restructuring Rural Credit in India. In: Antipode 42/1, 201-223.

Zadek, Simon (2013): Greening financial reform. https://www.project-syndicate.org/ commentary/integrating-the-green-growth-imperative-and-financial-market-reform-bysimon-zadek?barrier=accesspaylog (letzter Zugriff am 4.8.2019). 


\section{Southern cities in the context of climate- and development finance. Reregulation, disciplining and depoliticization.}

This paper examines how shifts in climate and development finance and related global action agendas become manifest at the urban scale. In recent years, the increasing focus of financial markets on environmentally sustainable projects has gone hand in hand with their heightened engagement in global development strategies. These entangled shifts have widened the scope of financial market activity, in terms of economic sectors but also geographically, particularly as global climate and development policies are increasingly targeting cities in so called „emerging markets“. Parts of economic geography (Bracking 2015a; Ouma/Johnson/Bigger 2018), development studies (Mader 2017; Mawdsley 2018), and environmental studies (Asiyanbi 2017; Bigger 2018) have critically discussed the related organizational, discursive, and regulatory shifts. Yet these literatures have only paid marginal attention to their urban dimension. Concomitantly, urban research has not sufficiently reflected the new relevance of Southern cities as actors, objects, and sites of intervention for climate and development agendas, which build on the deepening and expansion of global capital flows.

Our aim in this paper is to trace relevant dynamics in climate and development finance and to analyse their effects on cities and urban development. Theoretically, this paper builds on political economy and political ecology perspectives to show how the increasing entanglement of climate and development finance on the terrain of the city advances processes of reregulation, disciplining, and depoliticisation. Our conclusion proposes an agenda for future research. 